\title{
Management of a Perforating External Infection Related Resorptive
} Defect: A Case Report.

\author{
Hussain $\mathrm{SMI}^{1}$, Farzana $\mathrm{F}^{2}$, Chowdhury AFMA ${ }^{3}$, Mahmud $\mathrm{AA}^{4}$
}

\begin{abstract}
This case report describes the treatment of an external infection related root resorption in a maxillary left central incisor which perforated the root canal apical to the cervical area. Since the resorptive perforation was large with concomitant bony lesion, treatment with a combination of nonsurgical and surgical approach was chosen. After a surgical flap was reflected, the pathologic defect was curetted, root canal space was dried and the resorptive defect was repaired with light-cured glass ionomer cement. At the same visit, root canal filled with gutta percha by lateral condensation technique and the bony cavity was filled with calcium hydroxyappatite crystal. After 18 months, the patient remained asymptomatic and the tooth was functional.
\end{abstract}

Key Words: External Infection Related Resorption, root perforation, light-cured glass ionomer cement, hydroxyapatite crystal.

\section{Introduction}

External infection related resorption represents a combined injury to the pulp and periodontal ligament and where bacteria, primarily located in the pulp space and in dentinal tubules, trigger osteoclastic activity on the root surface. This type of resorption can affect all parts of the root. It is almost exclusively related to acute trauma and is especially common after intrusion and replantation of avulsed teeth. Pathogenesis lies in the event that the initial resorption has penetrated the cementum and exposed dentinal tubules, toxins from bacteria present in the dentinal tubules and/or the infected root canal can be diffused via the exposed tubules to the PDL. This results in continuation of the

01. Corresponding Author:

Professor Dr. S.M. Iqbal Hussain, BDS, DDS, MCPS

Professor of Conservative Dentistry \& Endodontics

Dhaka Dental College, Dhaka.

02. Dr. Farhad Farzana, BDS, FCPS (Trainee)

Honorary Medical Officer

Department of Conservative Dentistry \& Endodontics

Dhaka Dental College \& Hospital, Dhaka.

03. Dr. A.F.M. Almas Chowdhury, BDS, DDS

Assistant Professor of Conservative Dentistry \& Endodontics

Sapporo Dental College, Dhaka.

04. Dr. Abdullah Al Mahmud, BDS, FCPS (Trainee)

Honorary Medical Officer

Department of Conservative Dentistry \& Endodontics

Dhaka Dental College \& Hospital, Dhaka. osteoclastic process and an associated inflammation in the PDL, leading to resorption of adjacent alveolar bone ${ }^{1}$.The periodontal infiltrate consists of granulation tissue with lymphocytes, plasma cells and polymorphnuclear leucocytes. Multinucleated giant cells resorb the denuded root surface and this continues until the stimulus (pulp space bacteria) is removed. Radiographically, the resorption is observed as progressive radiolucent areas of the root and adjacent bone ${ }^{2}$.

Clinically, patient may remain asymptomatic or may present with increased mobility, dull pain and swelling. Sometimes the tooth may be extruded. Sensibility testing gives no response, and sometimes a sinus tract develops ${ }^{1}$.

In cases without a perforation, if bacteria are eliminated from the root canal and dentinal tubules by endodontic therapy, the resorptive process will be arrested. The resorption cavity will be then filled in with cementum or bone, according to the type of vital tissue found next to the resorption site (PDL or bone marrow-derived tissue) ${ }^{1}$.

However, in cases in which a pathway between the pulp canal space and the periodontal tissues is present, root canal treatment should be followed by repair of the perforation site with a suitable sealing material ${ }^{3}$. Repair may be instituted in one of two ways, either non-surgically by approaching the defect internally through the tooth or surgically by using an external approach through the periradicular tissues ${ }^{4}$.

If the lesion is located on the distal of lingual surface of the root, it may be impossible to visualize and correct the defect from a surgical approach. In these situations, intentional replantation for a single-rooted tooth or root amputation or hemisection for multi-rooted tooth may be the only treatment choices if an attempt is to be made to retain the tooth. Otherwise, extraction may be the only option ${ }^{5}$.

Indications for surgical intervention are as follows ${ }^{6}$.

- large perforations

- perforation as a result of resorption

- failure of healing after non-surgical repair

- non-surgically inaccessible perforations

- extensive coronal restorations

- when concomitant management of the periodontium is indicated and

- large overfilling. 
Before surgical intervention, the following parameters should be considered: ${ }^{6}$

- amount of remaining bone,

- extent of osseous destruction,

- duration of the defect,

- periodontal disease status,

- soft tissue attachment level,

- patient's oral hygiene, and

- surgeon's expertise in tissue management.

Many materials have been used to repair perforations, including amalgam, Cavit, Super ethoxybenzoic acid (super EBA), glass ionomer cement, and mineral trioxide aggregate (MTA) ${ }^{7}$ Other materials utilized included dentin chips, Biodentin, hydroxyapatite, rapid setting resin ionomer (Geristore), freeze dried bone, Plaster of Paris and Superbond $d^{8-10}$.

This report describes the treatment of a perforating external infection related resorptive defect following combination of non-surgical and surgical approach in a maxillary central incisor using light-cured glass ionomer cement.

\section{Case Report}

A 30-year old male presented with mild pain and extrusion in the left maxillary central incisor tooth. The patient was aware of occasional buccal swelling between the maxillary incisors for almost 2 months. There was a history of avulsion followed by replantation of the same tooth almost 20 years back. There was no history of endodontic therapy. The medical history of the patient was non-contributory. On examination, the maxillary central incisor exhibited mild extrusion and no discomfort was noted on percussion. On the mesiobuccal aspect of the crown, the probing depth measured $4 \mathrm{~mm}$, whereas the mobility of the tooth was within normal limits. Pulp sensibility test was negative. A periapical radiograph revealed a large radiolucency on the mesial root surface with a lateral root perforation and a lateral radiolucency on bone adjacent to the perforation (Figure 1). A small apical lesion with apical root resorption was also evident. The image of the lesion was shifted when additional radiographs were taken from different angulations.

The case was diagnosed as a perforating external infection related resorption in maxillary left central incisor tooth. Since the perforation was due to external root resorption and large enough, as well as the bony lesion was evident, at first a non-surgical root canal treatment followed by surgical repair of resorptive defect was planned. A combination of non-surgical and surgical approach of treatment plan was under consideration. The guarded nature of the prognosis was explained to the patient and consent obtained.

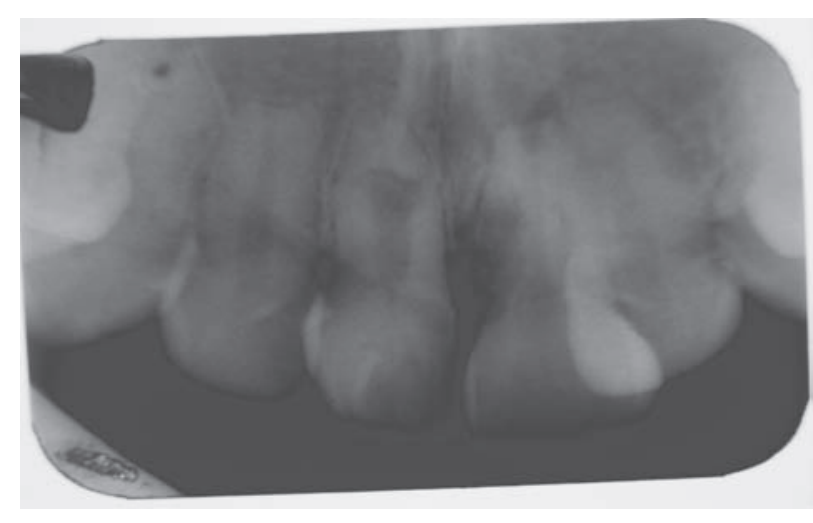

Figure 1: Initial $\mathrm{x}$-ray film showing external root resorption with periapical radiolucent lesions

At first visit an access cavity was prepared and the working length was determined by $\mathrm{x}$-ray images. The root canal was instrumented with stainless steel hand files, and frequently irrigated with normal saline. Use of a disinfectant irrigating solution such as sodium hypochlorite was not considered as the perforation was so large as to allow the irrigants to significantly damage the periradicular tissues. The persistent seeping of blood through the root canal diminished gradually with instrumentation. Subsequently, calcium hydroxide was placed as a temporary dressing to control bleeding. The access cavity was temporarily seal with zinc oxide eugenol cement. After 10 days, the root canal was reentered and irrigated alternately with sterile saline to remove the temporary dressing. But the canal could not be dried properly due to persistent seeping of blood, and then a combination treatment was decided.

With a combination treatment, under local anaesthesia a full-thickness mucoperiosteal flap was reflected extending from distal to the right maxillary central incisor to distal to the left maxillary lateral incisor. After surgical exposure and soft tissue debridement of the area revealed an extensive lesion involving the mesial-facial aspect of the root. The pathologic defect on the root surface was curetted the bony cavity was cleaned off and haemostasis was achieved by rinsing with sterile saline and pressure pack with gauze (Fig- 2).

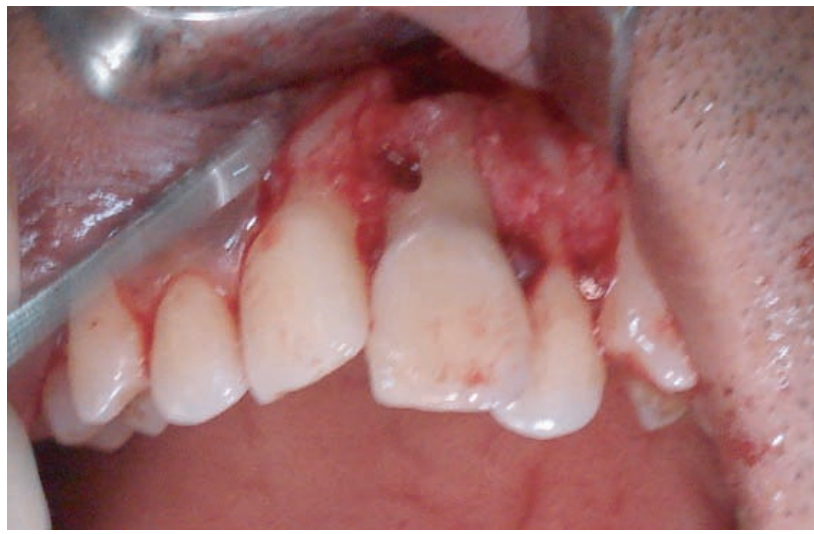

Figure 2: Perforation on lateral and facial aspect of root. 
The root canal space was dried and a tight fitting gutta percha point without sealer was placed into the root canal space to prevent blockage of the canal. The perforation defect was then repaired using a light-cured glass ionomer (GC Fuji Lining LC, Japan), following the manufacturer's instruction. Before placing restoration on the defect, a dentin conditioner was applied to the dentin surrounding the perforation and left in place for 10 seconds. This was followed by rinsing with distilled water for five seconds and lightly drying with air and a sterile cotton pellet. The material was mixed to a slightly running consistency for ease of application and was carried into place by using a periodontal probe. The perforation site was gradually filled up from the base with the material covering about 2-3 mm of the surrounding dentin and then light-cured (Figure 3). Final thickness of the material was about $2 \mathrm{~mm}$.

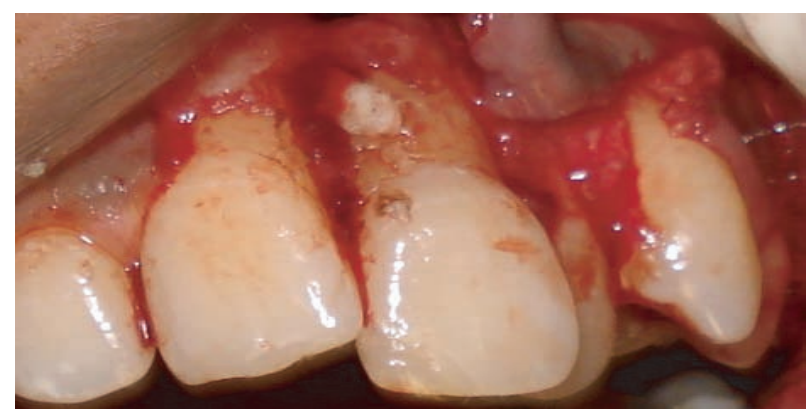

Figure 3: Light-cured glass ionomer repair of External Resorptive Perforation .

After the initial set of the repair material, the gutta-percha point was removed and the internal root canal owas completed using gutta percha and zinc-oxide sealer in lateral condensation method. At the same time, the apical lesion was curetted and a retrofilling with glass also placed and a post operative X-ray was obtained ( Fig 4).

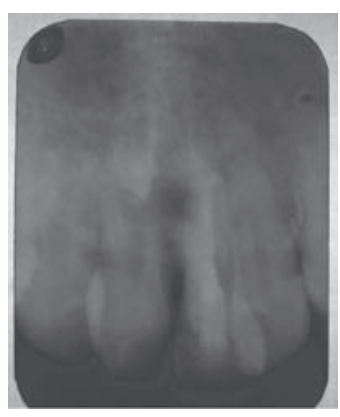

Figure 4: Periapical X-ray film showing obturation and repair of perforation.

The bony cavity was then filled with calcium hydroxyapatite crystal and the flap was retracted and sutured (Figure 5). Ibuprofens $400 \mathrm{mg} 3$ times a day orally for 3 days, as well as amoxicillin $500 \mathrm{mg} 3$ times a day orally for 7 days were given. Also, $0.2 \%$ chlorhexidine gluconate mouth rinse was prescribed for 7 days. The patient was recalled 1 week later for suture removal.

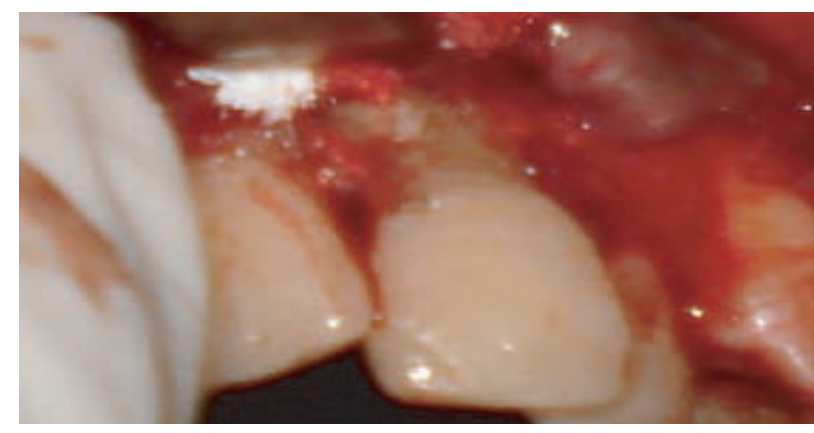

Figure 5: Hydroxyapatite crystal on bony cavity.

At the next visit, the patient reported no postoperative pain or discomfort. The first follow-up was planned at the postoperative sixth month. Subsequent controls were planned as 12-month intervals. However, the patient moved to another city and could not be controlled anymore because of this relocation. After 18 months, he reported over telephone that he experienced no pain or swelling during this period.

\section{Discussion}

External Infection-Related Resorption entity was described in a clinical and histologic study in 1965 of avulsed and replanted teeth. This resorption is a rapidly progressing process that may result in total resorption of the root within a few months. It is often very difficult to distinguish external from internal root resorption. It has been suggested that diagnosis should always be confirmed while the treatment is proceeding ${ }^{1}$.

Since resorptive defects are often asymptomatic, they are usually recognized by routine radiographs. However, in this case, the patient recognized the occasional swollen gingiva between maxillary central incisors, which prompted him to seek treatment.

This type of resorption represents a combined periodontal and pulpal injury and requires immediate endodontic treatment to control or remove the osteoclast promoting factors (bacterial toxins from the root canal system). The progressive nature of this type of root resorption has been associated with an ongoing inflammation from a source of infection $^{1}$. In this case, history of avulsion and replantation could be regarded as a source of cemental damage and bacterial infection to pulp cavity.

External root resorption lesions may or may not communicate with the pulp canal space. However, in this case, the mesial root wall was perforated at mid root level apical to the cervical area. Diagnosis of the resorptive defect on facial or lingual/palatal surface was missed during radiographic examination. Use of Tuned Aperture Computed Tomography (TACT) or Cone Beam Computed Tomography (CBCT) could not be considered due to unavailability ${ }^{2}$.

Many materials have been used to repair perforations including amalgam, Cavit, Super EBA, glass ionomer cement, and MTA. In addition to providing a good seal, the 
materials for repair of perforations must be biocompatible, nontoxic, insoluble in the presence of tissue fluids, and capable of promoting regeneration of the periradicular tissues. Based on the outcome of some studies, MTA is an excellent material for the repair of perforations at various levels of the root. However, one of the limitations of MTA is its extended setting time and difficulty in handling. The material is more suitable for box-like cavities where it can be lightly packed. This is a drawback not only for potential users but for experienced operators but for experienced operators as well ${ }^{9}$.

In clinical situations when this material is unavailable, clinicians are forced to resort to other materials when trying to save a perforated tooth. The material of choice must exhibit basic properties such as biocompatibility, ability to adhere to tooth structure for adequate sealing and ease of application $^{8}$. In this case report, glass ionomer cement was chosen as an alternative to MTA due to economic consideration.

In this case report, a light-cured glass ionomer was chosen as an alternative to MTA. Light-cured glass ionomer is a small particle, hydrophilic, non-aqueous resin combined with a photo initiator and glass powder formulation. The advantages of this material are its insolubility in oral fluids, reasonable adhesion to tooth structure, high strength, and dual cure properties. Glass ionomers also offer the following attributes: low shrinkage, low thermal expansion and extended fluoride release. Clinical and histological investigation of glass ionomer demonstrates a biocompatibility to both soft and hard tissues. The formation of an epithelial and connective tissue adherence to light-cured glass ionomer represents a significant advancement in the ability to restore previously considerded hopeless teeth. As an additional benefit, fluoride release from glass ionomer may positively affect bacterial plaque biochemistry through an alteration of carbohydrate metabolism. Its setting is relatively fast and thus improving performance reducing messy handling and so less demanding 8 .

In majority of the previous studies ${ }^{3}$ the root canal filling has been placed after repair of the perforation defect. On the other hand, Altundasar and Demir, Yildirim ${ }^{3}$ has repaired the perforation after the root canal filling has been completed. In this case, we also preferred repair of the perforation and then root canal filling. The inherent danger with this technique is that the compacting forces required to perform the obturation procedure may displace the external repair material ${ }^{3}$. In this case, repair material was undisturbed because of careful handling. Subsequently, the bony cavity was filled with calcium hydroxyappatite crystal, an alloplastic material as studies showed reduction of the osseous defects greater in hydroxyappatite than curettage only ${ }^{10}$.
Though there was no bone covering the root surface coronal to the defect, a periodontal regenerative procedure could not be performed in conjunction with the corrective surgical procedure due to non-availability.

Based on biologic compatibility, economically inexpensive and availability, light-cured glass ionomer material may be considered to be part of the clinician's armentarium for the treatment of endodontic perforations, especially when moresuitable materials such as MTA are unavailable. However, more evidence from randomized controlled clinical trials needs to be generated to assess whether a more conclusive valid recommendation can be made about the performance of light-cured glass ionomers for the repair of endodontic perforations.

\section{References}

1. Ove J, Andreasen, Bakland LK. Pathologic tooth resorption. In: Ingle JI, Bakland LK, Braumgartner C. Ingle,s Endodontics.6th ed. Hamilton,BC Decker Inc; 2008;1358-1380.

2. Trope M. Root resorption due to dental trauma. Endodontic Topics. 2002;1:79-100.

3. Altundasar E, Demir B. Management of a Perforating Internal Resorptive Defect with Mineral Trioxide Aggregate: A Case Report.

4. Roda RS, Bradley H, Gettleman. Non-surgical Retreatment. In: Cohen S, Kenneth M, Hargreaves. Pathways of the pulp. 9th ed. Elsevier New Delhi, 2006; 944-1005.

5. Gerald N, Glickman, Hartwell GR. Endodontic Surgery. In: Ingle JI, Bakland LK, Braumgartner C. Ingle,s Endodontics. 6th ed. Hamilton, BC Decker Inc; 2008;233-1294.

6. Tsesis I and Fuss Z. Diagnosis and treatment of accidental root perforations. Endodontic Topic, 2006;13, 95-107.

7. Zhang C, Chan AW, Lahmal W. Mineral Trioxide Aggregate repair of lateral root perforation using intentional replantation and bone grafting. Hong Kong Dent J 2011;8: 51-55.

8. Farea. Endodontic perforation repair with light cured glass ionomer. International Dentistry-African edition: 2008; $1: 84-92$.

9. Bronecce F. BioDentine: A dentin substitute for the repair of root perforations, apexification and retrograde root filling. Master thesis (Biomaterial Sciences). . Université de Paris,13.2009.

10. Bashutski JD, Wang HL. Periodontal and Endodontic Regeneration. Journal of Endodontics, 35;3:2009. 